\title{
MSD OFFICE BUILDING DESIGN OF INTELLIGENT LIGHTING
}

\section{SYSTEM}

\author{
Jiangtao Xing ${ }^{1}$, Yuchen Chen $^{2}$ \\ College of Electronics and Electrical Engineering, Shanghai University of Engineering Science, Songjiang District, \\ Shanghai 201600, China \\ College of Electrical and Electronic Engineering, Shanghai University of Engineering Science, Songjiang District, \\ Shanghai, China \\ 1jiangtaoxing@sina.com \\ 2yuchen1957@163.com
}

\begin{abstract}
This will be the MSD Asia Pacific head-quarters and headquarters office building decoration project of China, introduces the intelligent lighting system design of office buildings. Intelligent design considerations of lighting system is controllable change of indoor illumination, interior illumination changes controlled by a dimmer system as a whole. One of the main components for mobile and presence sensors and illumination sensors, through the identification of people and its surrounding light intensity measurements, adjust the output light source lamp, light intensity and stability of the entire space within a certain range.
\end{abstract}

Keywords: Intelligent Lighting Control System For Office Building Illumination Sensor

\section{INTRODUCTION}

Present office building's lighting system mostly using the traditional control method: lamps are controlled directly by the switch; the luminous flux of the light output fixed. This lighting system is rigid, there are mainly the following points:

1. Lightingtechnology is backward. Inefficient use of light sources led lighting energy consumption.

2. Lighting design is not reasonable. Mainly tend to the lighting, lighting power a waste of energy.

3. Lighting maintenance management level is not easy and unable to control lighting switches, a lack of effective management.

Use intelligent lighting system can solve these problems, it has the following advantages:

1. The automatic control of lighting

2. Beautifying the environment

3. Extended lamp life

4. Save energy
5. Consistent illumination

6. Easy to control

The actual application of this design is the world's leading biopharmaceutical company MSD or Merck (Chinese MSD) Asia-Pacific headquarters and headquarters office building decoration project of China electrical and mechanical parts of the lighting system. This design has the three most basic requirements:

And guarantee the most basic lighting work;

Second, lighting to achieve the desired result;

Third, the high efficiency, energy saving lighting system.

The design of the final result will be verified upon completion of the project.

\section{INTELLIGENT LIGHTING SYSTEM CONTROL PROCESS}

Intelligent lighting control systems, lighting using lamps of high efficiency, energy-saving electric light source (LED 
lamps are commonly used), and at the same time using intelligent control functions (this stage is adjustable rectifier). Intelligent control system of lighting signal input of the sensor area to intelligent control modules, and then given a fixed output value (the luminous flux of the lamp output is fixed), so as to achieve control of the illumination within the region. Control flow diagram is shown in Figure 1:

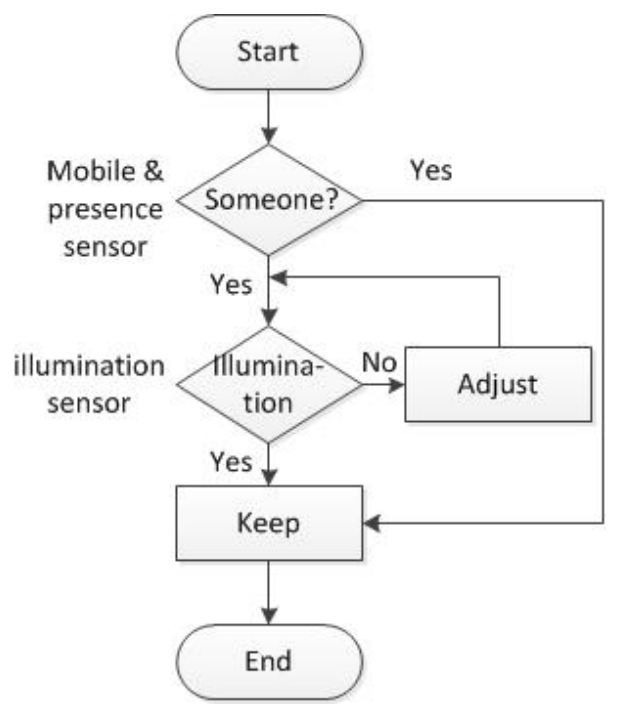

Figure 1. flow chart

Office building of intelligent lighting control system is based on environmental characteristics, and whether it was judged, light intensity in the region followed by, according to the set working status, automatically switching between the basic working conditions, reached the objective of energy saving and efficient. Before coming to work for half an hour, the system will automatically turn on and around the building room by the window will make the best use of outdoor light, lamp brightness automatically dims sunny weather, rainy weather in lamp brightness is automatically adjusted to keep the room illumination balance. And when the end of the day, after open system after a set time "rest" mode, automatic gradually extinguished lights in each region. Meanwhile, infra-red detection capability of the system to open, no man's land of LEDs are all off; it was overtime, adjust the brightness of the light, the illumination of the region is in the appropriate range.

Meanwhile, the system can also be manually controlled (programmable switch panels), wireless remote control (remote control) Regulation of light intensity. Such as lunch time by switch panel control lamps, curtains make the room reaches a comfortable environment and in special areas such as conference rooms or indoor light intensity can be adjusted according to different needs.

\section{CONTROL PROGRAMMES}

As a component of the intelligent control system, field detection devices--sensor plays a very important role, because of the different Office environment, light angle, caused by different light intensity sensor options will directly influence the dimming of lamps to choose, which had an impact on lighting energy-saving effect. Here's this used to human presence and moving wave sensors and illumination sensors as the core of intelligent lighting control system.

Intelligent lighting control system for this project using the system C-BUS, he is a distributed intelligent control system, bus type, mainly for lighting and curtains, projector, motorized screen, air conditioning control linkage system.

System components are built-in microprocessor and memory units, consists of a pair of signal cables connected to a network. Each unit sets a unique address using software to set the function of the unit, control loop through output unit. Enter the unit through the group address and output components for the corresponding contact. When there is input, the input element into it C-BUS signal broadcast over the C-BUS system bus, judging from all the output units receive signals and control circuit output (the luminous flux of the lamp). The schematic diagram is shown in Figure 2:

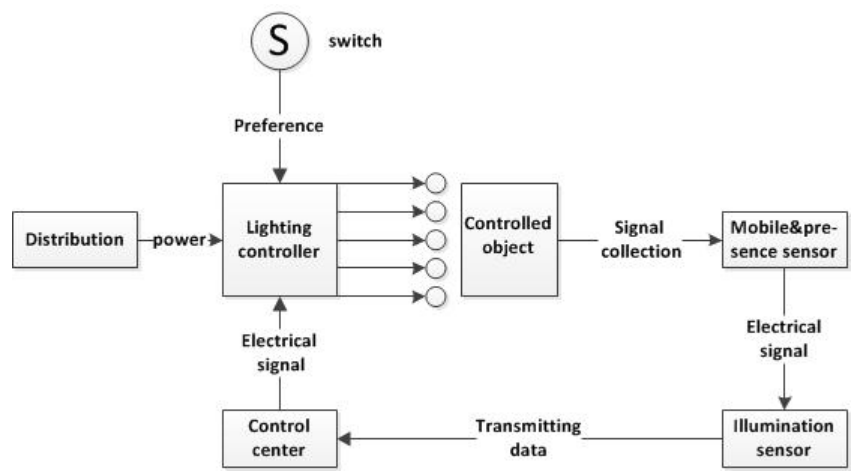

Figure 2. control system architecture diagram 


\section{CONTROL CHARACTERISTICS}

Intelligent control system of the C-BUS is characterized by its presence and use of mobile sensor, this sensor infrared sensor and ultrasonic sensor type. Infra-red sensor is the body of Pyroelectric infrared sensor PIR detection of infrared wavelengths of the human body to sense the movement of people and judging people, apply to people in smaller areas. Ultrasonic sensors are sound waves generated by induction of human motion identification of human existence, applies to the area of the large flow of people. At present, the Office building is to use human Pyroelectric infrared sensors, this sensor has the following disadvantages:

1, are vulnerable to a variety of heat, light interference;

2, can not easily be received;

3 , under the influence of the ambient temperature.

Ultrasonic sensor for other creatures (mice) to identify less effective. In order to increase the sensitivity of the sensor is used with ultrasonic infrared double standards. Therefore, the system uses a Pyroelectric infrared sensors and acoustic sensor combination of movement and presence sensor can distinguish the good indoor existence.

Each lamp has a dimmer function of indoor, lighting can be in the Office of the control panel and remote master control, environment (including illumination, infrared and acoustic) testing is done by the existence and motion sensor and light sensor. Illumination requirements for General Office for 300 4001x, you can set the upper and lower values for the system (certain areas, depending on the circumstances may be). When it detects Office and in the Office when the illumination is not within the range, will be based on the average illuminance calculation formula

$$
\begin{aligned}
& E=\frac{\phi N U K}{S} \\
& \text { E-------average illuminance } \\
& \Phi \text {------light flux } \\
& \mathrm{N} \text {-------number of light sources }
\end{aligned}
$$

Utilization coefficient of U-------

K-------luminaire maintenance factor

S--------area
System will adjust the brightness of the lamps, and then adjust the illumination of offices, Office began keeping in the context of setting of illumination. And if no one in the Office or Office illumination setting range, the system will maintain the current status. But when it comes to time off, no overtime area lights will slowly go out; some overtime region is maintain a normal working levels.

\section{DESIGN RESULTS}

In Figure 3, for the lighting of a room loop map

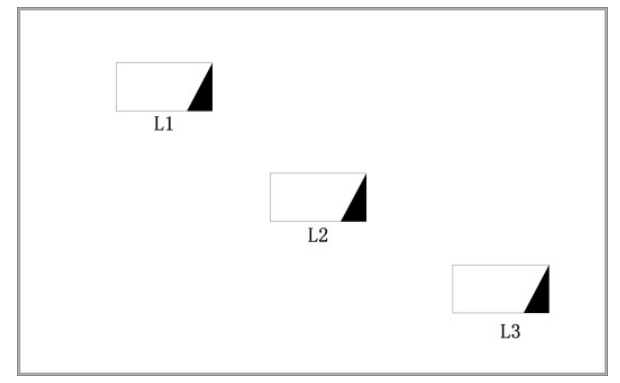

L1, L2, L3, respectively, in the Chamber of representatives of the three lighting circuits, three circuits of power respectively, $0.8 \mathrm{Kw}, 0.75 \mathrm{Kw}, 0.6 \mathrm{Kw}$. According to the General working time, before going to work in lighting time period $0.5 \mathrm{H}$ to $0.5 \mathrm{H}$ after work, for about $10 \mathrm{H}$.

Using traditional lighting system, its power is:

$W_{1}=\sum P * t$

$=0.8 * 10+0.75 * 10+0.6 * 10=21.5 \mathrm{Kwh}$

Intelligent lighting system, sunny days average power of three circuit respectively, $0.65 \mathrm{Kw}, 0.7 \mathrm{Kw}, 0.45 \mathrm{Kw}$, whose power is:

$W_{2}=\sum P * t$

$=0.65 * 10+0.7 * 10+0.45 * 10=18 \mathrm{Kwh}$

Cloudy or rainy days when the average power of the three circuits respectively, $0.73 \mathrm{Kw}, 0.72 \mathrm{Kw}, 0.55 \mathrm{Kw}$,

Its power consumption is

$W_{3}=\sum P * t$

$=0.73 * 10+0.72 * 10+0.55 * 10$

$=20 \mathrm{Kwh}$

Sunny percent power savings

$\rho_{1}=\left(W_{1}-W_{2}\right) / W_{1} * 100 \%$

$=(21.5-18) / 21.5 * 100 \%$

$=16 \%$

Cloudy or rainy percent power savings 
$\rho_{2}=\left(W_{1}-W_{3}\right) / W_{1} * 100 \%$

$=(21.5-20) / 21.5 * 100 \%$

$=7 \%$

\section{CONCLUSION}

Through the design of the system, compared with the traditional building, energy saving in lighting at $7 \% \sim 16 \%$, office buildings and lighting effects are also more obvious, intelligent control of its effect. Therefore, this kind of lighting design ideas can be applied to a variety of related sites.

\section{REFERENCES}

[1] Tang Hai,Construction electricity design and construction [M]. Beijing: China architecture and building press, 2007

[2] $\mathrm{Xu}$ Jinbiao, Zhang Zhenzhao. Intelligent buildings technologies [M].Third Edition. Beijing: China machine press, 2010

[3] Wu Peng.Pyroelectric sensor networks based on wireless tracking systems of the human body [D].Wuhan. Wuhan University of technology, 2012

[4] Cui Yanqin. Based on Pyroelectric human object recognition system for wireless sensor networks[D]. Harbin. Harbin Institute of technology, 2014

[5]Zhong Ling Yu, Wang Li. Discussion on energy saving measures of architectural lighting $[\mathrm{J}]$. architectural design management 2009 (03)

[6]Valerio R.M. Lo Verso,Anna Pellegrino,Franco Pellerey.A multivariate non-linear regression model to predict the energy demand for lighting in rooms with different architectural features and lighting control systems[J]. Energy and Buildings, 2014, 76:151-163

[7]Jiao Quanhu.Intelligent building and building energy saving[J].information technology, 2009 (03)

[8] Su Zhu. intelligent lighting system [D]. Jinan. Shandong University, 2012

[9] Liu Hao.Office lighting control strategies based on natural lighting and energy saving analysis[D]. Xian. Xian University of architecture and technology, 2010

[10]Liu Yaning.Study on the daylighting and energy consumption of office building[D]. Tianjin. Tianjin University, 2008 